\title{
Glycosaminoglycans of human rotator cuff tendons: changes with age and in chronic rotator cuff tendinitis
}

\author{
G P Riley, R L Harrall, C R Constant, M D Chard, T E Cawston, B L Hazleman
}

\begin{abstract}
Objectives-To analyse the glycosaminoglycans of the adult human rotator cuff tendon matrix, to characterise changes in the glycosaminoglycan composition with age and in chronic rotator cuff tendinitis.

Methods-Rotator cuff (supraspinatus) tendons $(n=84)$ and common biceps tendons $(n=26)$ were obtained from cadavers with no history of tendon pathology (age range 11-95 years). Biopsies of rotator cuff tendons (supraspinatus and subscapularis tendons, $\mathbf{n}=53$ ) were obtained during open shoulder surgery to repair shoulder lesions (age range 38-80 years). Glycosaminoglycans were extracted by papain digestion and analysed by cellulose acetate electrophoresis, the carbazole assay for uronic acid and the dimethylmethylene blue dye-binding assay for sulphated glycosaminoglycans. Some digests were analysed for keratan sulphate by 5D4 monoclonal antibody ELISA. Soluble proteoglycans were extracted in $4 M$ guanidine hydrochloride and analysed by 4-15\% SDS PAGE.
\end{abstract}

Results-The mean (SD) sulphated glycosaminoglycan (GAG) content of the normal cadaver supraspinatus tendon was $12 \cdot 3(4 \cdot 3) \mu \mathrm{g} / \mathrm{mg}$ dry weight, between three and ten times greater than in the common biceps tendon $[1.2(0.6) \mu \mathrm{g} / \mathrm{mg}$ dry weight]. The major GAG was chondroitin sulphate $[6.9(2 \cdot 6) \mu \mathrm{g} / \mathrm{mg}$ dry weight], with a smaller proportion of dermatan sulphate $[2.5(1.2) \mu \mathrm{g} / \mathrm{mg}$ dry weight]. In contrast, the common biceps tendon contained predominantly dermatan sulphate $[0.8(0.2) \mu \mathrm{g} / \mathrm{mg}$ dry weight] with less chondroitin sulphate [0.2 (0.2) $\mu \mathrm{g} / \mathrm{mg}$ dry weight]. There was no difference in the concentration of hyaluronan in these tendons [9.3 $(2 \cdot 8)$ $\mu \mathrm{g} / \mathrm{mg}$ dry weight and $10.8(4 \cdot 3) \mu \mathrm{g} / \mathrm{mg}$ dry weight respectively] and there was no significant change of hyaluronan with age. Keratan sulphate was a small but significant component of the supraspinatus tendon $[0.43(0.33) \mu \mathrm{g} / \mathrm{mg}$ dry weight, $n=25$ ], whereas there was little or none in the common biceps tendon [0.04 $(0.05) \mu \mathrm{g} / \mathrm{mg}$ dry weight, $n=8$ ] and there was no significant change across the age range. In the supraspinatus tendon, there was a significant decrease in total glycosaminoglycan, chondroitin sulphate and dermatan sulphate with age $(p<0.001)$, whether expressed relative to the tendon dry weight or total collagen content, and no change in the relative proportion of the different GAG types. There was, however, a large degree of variation within the samples. Supraspinatus tendons from patients with chronic tendinitis had a significantly increased concentration of hyaluronan [30.4 (10.1) $\mu \mathrm{g} / \mathrm{mg}$ dry weight, p < 0.001], chondroitin sulphate $[8.4(1.8)$ $\mu \mathrm{g} / \mathrm{mg}$ dry weight, $p<0.05]$ and dermatan sulphate $[3.8(1.1) \mu \mathrm{g} / \mathrm{mg}$ dry weight, p < 0.001] compared with normal cadaver supraspinatus tendons, although the keratan sulphate content was not significantly different $[0.18(0.05) \mu \mathrm{g} / \mathrm{mg}$ dry weight].

Conclusions-The normal supraspinatus tendon has the proteoglycan/glycosaminoglycan of tendon fibrocartilage, which it is suggested is an adaptation to mechanical forces (tension, compression and shear) which act on the rotator cuff tendons in the shoulder, although other factors such as reduced vascularity, low oxygen tension and the influence of local growth factors may also be important. This functional adaptation may have important consequences for the structural strength of the supraspinatus tendon and to influence the ability of the tendon to repair after injury. The glycosaminoglycan composition of tendon specimens from patients with chronic tendinitis is consistent with acute inflammation and new matrix proteoglycan synthesis, even in relatively old tendon specimens and after at least one injection of corticosteroid.

(Ann Rheum Dis 1994; 53: 367-376)

Chronic shoulder pain is a common and disabling condition that is often seen in rheumatological practice and prevalent in the elderly community. ${ }^{1}{ }^{2}$ Relatively little is known about the underlying pathology, as surgical biopsies of human tendons are rarely obtained and are not required for diagnosis. The main causes of shoulder pain are lesions of the rotator cuff tendons, most commonly the supraspinatus tendon, although the subscapularis, infraspinatus and teres minor 
tendons may also be involved. ${ }^{3}$ The clinical description of 'tendinitis' is often used to describe painful tendon lesions, although there is little evidence for an acute inflammatory process in degenerate and spontaneously ruptured tendons. Rotator cuff tendinitis does not necessarily resolve with time and is often refractory to conservative therapies, including rest, physiotherapy and local corticosteroid injections. ${ }^{45}$

Degeneration of the tendon matrix is generally considered to predispose to 'tendinitis' and eventual rupture, as normal tendons are immensely strong under tension. ${ }^{6}$ A variety of factors including ageing, vascular insufficiency, the anatomical shape of the acromium, impingement against osteophytes, and repetitive activities have all been implicated in the pathology of rotator cuff tendinitis. $^{78}$ Whatever the cause, the most frequent site of all tendon lesions is the "critical zone' in the supraspinatus tendon, approximately one centimetre from the bone insertion and a region of blood vessel anastomoses. ${ }^{9}$

Although they form a minor proportion of the extracellular matrix, proteoglycans and their constituent glycosaminoglycans can influence many important physiological processes, such as ion transport, the diffusion of nutrients and water retention. ${ }^{10}$ They are also involved in specific interactions that can influence important processes such as collagen fibrillogenesis, cell-cell interactions, growth factor binding and cell regulation. ${ }^{11}{ }^{12}$ Tendons have previously been considered to be relatively inert and homogenous structures, although there are regional differences within tendons and differences in structure and composition between different tendons. ${ }^{13}$ In particular, changes in the glycosaminoglycan and proteoglycan composition have been described during maturation and ageing, ${ }^{14}$ after immobilisation and exercise ${ }^{15}$ in response to mechanical forces ${ }^{16}$ and after tendon injury. ${ }^{17}$

A large histopathological study of 865 spontaneously ruptured human tendons (not including rotator cuff tendons), described an accumulation of glycosaminoglycans between the collagen fibres in $21 \%$ of tendons. ${ }^{18}$ These degenerative changes were second in frequency only to cell changes ascribed to tissue hypoxia, which were found in $44 \%$ of tendons. Interstitial glycosaminoglycan accumulation was also observed in $9 \%(42 / 445)$ of non-ruptured tendons. In a recent histological study of macroscopically normal cadaver supraspinatus tendons, we showed that glycosaminoglycan accumulation was the most common abnormality, found in $18 \%$ of non-ruptured specimens but only in specimens over the age of thirty years. ${ }^{19} \mathrm{We}$ hypothesised that quantitative and qualitative changes in the matrix glycosaminoglycan and proteoglycan composition may be found in ageing and degenerate tendons and may predispose to chronic rotator cuff tendinitis. We investigated whether the ageing supraspinatus tendon is capable of maintaining the normal proteoglycan matrix and compared the glycosaminoglycan composition with a sample of common biceps tendons, a flexor tendon that is rarely involved in any pathology and likely to show changes due to ageing alone. We also obtained a relatively large number of surgical specimens of rotator cuff tendons from patients undergoing shoulder surgery for chronic tendinitis (a rotator cuff tear) and other painful shoulder conditions, to investigate the changes in glycosaminoglycans associated with rotator cuff tendinitis and human tendon pathology.

\section{Methods}

TENDON COLLECTION

Specimens of supraspinatus tendons and common biceps tendons were collected from cadavers at necropsy. None of the subjects had any known history of tendinitis or shoulder pathology. Shoulders with gross rotator cuff lesions were excluded from the study. Surgical biopsies of rotator cuff tendons (supraspinatus and subcapularis tendon) were collected by CRC. Tendon tissue was taken only when ethically justified, in order to bring together less damaged tissue to enhance the prospects of successful repair of sutured material. Patients with chronic 'tendinitis' (a rotator cuff tear) had been treated conservatively with at least one corticosteroid injection before surgery. Tendons with 'calcifying tendinitis' had radiographically detectable mineral deposits-tissue was excised from around the deposit. Biopsies of tendons were also taken during fracture repairs, prosthetic shoulder replacement and 'stabilisation' operations to prevent recurrent dislocation.

TENDON DISSECTION AND PROCESSING

Tendon specimens were dissected free of muscle, fat and all remains of the subacromial bursa and surrounding connective tissues. The specimen was freeze dried and powdered in a Spex freezer mill and stored desiccated at $-20^{\circ} \mathrm{C}$.

\section{GLYCOSAMINOGLYCAN ANALYSIS}

Tendon tissue was digested in $0.1 \mathrm{M}$ phosphate buffer $\mathrm{pH} 7 \cdot 0$ containing $125 \mu \mathrm{g} / \mathrm{ml}$ of papain, $10 \mathrm{mM}$ cysteine $\mathrm{HCl}$ and $2 \mathrm{mM}$ EDTA. The collagen content was calculated by hydroxyproline analysis, using a microtitre plate modification of the method of Bergman and Loxley. ${ }^{20}$ Sulphated glycosaminoglycans (GAG) were analysed by a microtitre plate modification of the dimethylmethylene blue assay, with and without specific glycosidase digestion with chondroitinase AC. ${ }^{21}$ Briefly, glycosaminoglycan extracts were digested for 18 hours at $37^{\circ} \mathrm{C}$ with $0.05 \mathrm{U} / \mathrm{ml}$ of enzyme. The proportion of chondroitin sulphate (C4S and $\mathrm{C} 6 \mathrm{~S}$ ) and dermatan sulphate (DS) was calculated using the following formulae:

Total GAG - GAG after

$\% \mathrm{CS}=\underline{\text { chondroitinase } \mathrm{AC} \text { digest }} \times 100$ 
$\% \mathrm{DS}=$

GAG post chondroitinase $A C$ digest -

GAG post chondroitinase $A B C$ digest Total GAG

Uronic acid (UA) was measured using a microtitre plate modification of the carbazole assay ${ }^{22}$ as described by S Carney (personal communication). The hyaluronan (HA) content was calculated assuming that UA comprised $36 \%$ of the dry weight of GAG and $45 \%$ of the dry weight of $\mathrm{HA},{ }^{23}$ using the formula:

$$
\mathrm{HA}=\frac{\mathrm{UA}-(\text { Total GAG } \times 0.36)}{0.45}
$$

The keratan sulphate content of some specimens was measured using the 5D4 monoclonal antibody ELISA. ${ }^{24}$ Cellulose acetate electrophoresis of papain digests was conducted for 1 hour at $45 \mathrm{~mA}$ in $0.3 \mathrm{M}$ calcium acetate $\mathrm{pH} 7 \cdot 2$. Glycosaminoglycans were stained with $0.2 \%$ Alcian blue $8 \mathrm{GX}$ in $0.3 \%$ acetic acid containing $0.05 \mathrm{M}$ magnesium chloride.

\section{PROTEOGLYCAN ANALYSIS}

Proteoglycans were extracted from powdered tendon for 48 hours at $4^{\circ} \mathrm{C}$ with $4 \mathrm{M}$ guanidine $\mathrm{HCl}$ in $0.05 \mathrm{M}$ sodium acetate $\mathrm{pH} 5.6$ containing the proteinase inhibitors $0.01 \mathrm{M}$ EDTA, 0.1 M 6-amino-caproic acid and 0.05 $M$ benzamidine $\mathrm{HCl}$. After centrifugation (15 $000 \mathrm{~g}$ for 10 minutes), the pellet was reextracted for 24 hours and the supernatants were pooled and stored at $-20^{\circ} \mathrm{C}$. Soluble proteoglycans were precipitated overnight at $-20^{\circ} \mathrm{C}$ with 9 volumes of ethanol containing $1 \%$ potassium acetate and taken up in $5 \times$ SDS-PAGE sample buffer. SDS-PAGE was conducted as described by Laemmli, ${ }^{25}$ using the Bio-Rad Mini-Protean II system and preformed $4-15 \%$ gradient gels. Proteoglycans were stained with Alcian Blue $8 \mathrm{GX}$ as described by Wall and Gyr. ${ }^{26}$ After extensive washing in water and destain to remove SDS, the gel was stained overnight with $0 \cdot 2 \%$ Alcian Blue $8 \mathrm{GX}$ in $3 \%(\mathrm{v} / \mathrm{v})$ glacial acetic acid containing $0.05 \mathrm{M}$ magnesium chloride and destained in $3 \%(\mathrm{v} / \mathrm{v})$ acetic acid, $0.05 \mathrm{M}$ magnesium chloride. Proteins were stained with $0 \cdot 25 \%$ Coomassie Brilliant Blue G250 in $40 \%$ methanol and $10 \%$ acetic acid.

Table 1 Specimens of adult human tendons

\begin{tabular}{llll}
\hline Tendon specimens & Observations & $\begin{array}{l}\text { Number of } \\
\text { specimens }\end{array}$ & $\begin{array}{l}\text { Mean age in years } \\
\text { (range) }\end{array}$ \\
\hline Supraspinatus (cadaver) & macroscopically 'normal' & 62 & $58 \cdot 1(11-95)$ \\
& 'ruptured' tendons & 11 & $81 \cdot 2(60-94)$ \\
& 'thin/degenerate' tendons & 4 & $78 \cdot 7(67-86)$ \\
& 'calcified' tendons & 5 & $82 \cdot 0(76-94)$ \\
Common biceps (cadaver) & misc abnormalities & 3 & $57 \cdot 3(43-84)$ \\
Supraspinatus biopsy & macroscopically 'normal' & 26 & $57 \cdot 6(12-83)$ \\
& 'tendinitis' (cuff tear) & 26 & $59 \cdot 5(38-80)$ \\
Subscapularis biopsy & 'calcifying tendinitis' & 5 & $52 \cdot 2(47-63)$ \\
& fracture repair & 2 & $51 \cdot 5(25$ and 78$)$ \\
& 'tendinitis'(cuff tear) & 8 & $73 \cdot 4(68-80)$ \\
& stabilisation operation & 5 & $25 \cdot 2(19-31)$ \\
& prosthetic replacement & 7 & $57 \cdot 3(40-76)$ \\
\hline
\end{tabular}

\section{MATERIALS}

Papain $(2 \times$ crystallised $)$ and glycosaminoglycan standards of chondroitin-4-sulphate, chondroitin-6-sulphate, dermatan sulphate, heparan sulphate, keratan sulphate and hyaluronan were purchased from Sigma, Poole, Dorset, UK. Chondroitinase AC, chondroitinase $\mathrm{ABC}$ and keratanase were obtained from ICN/Flow. Cellulose acetate membranes $(0.45 \mu \mathrm{m})$ were from Schleicher and Stull, Germany. All other reagents were analytical grade from FSA, Loughborough, UK.

STATISTICAL ANALYSIS

The difference between sample group means was tested for statistical significance by the Mann-Whitney rank sum test. Sample means were considered significantly different if $\mathrm{p}<0.05$.

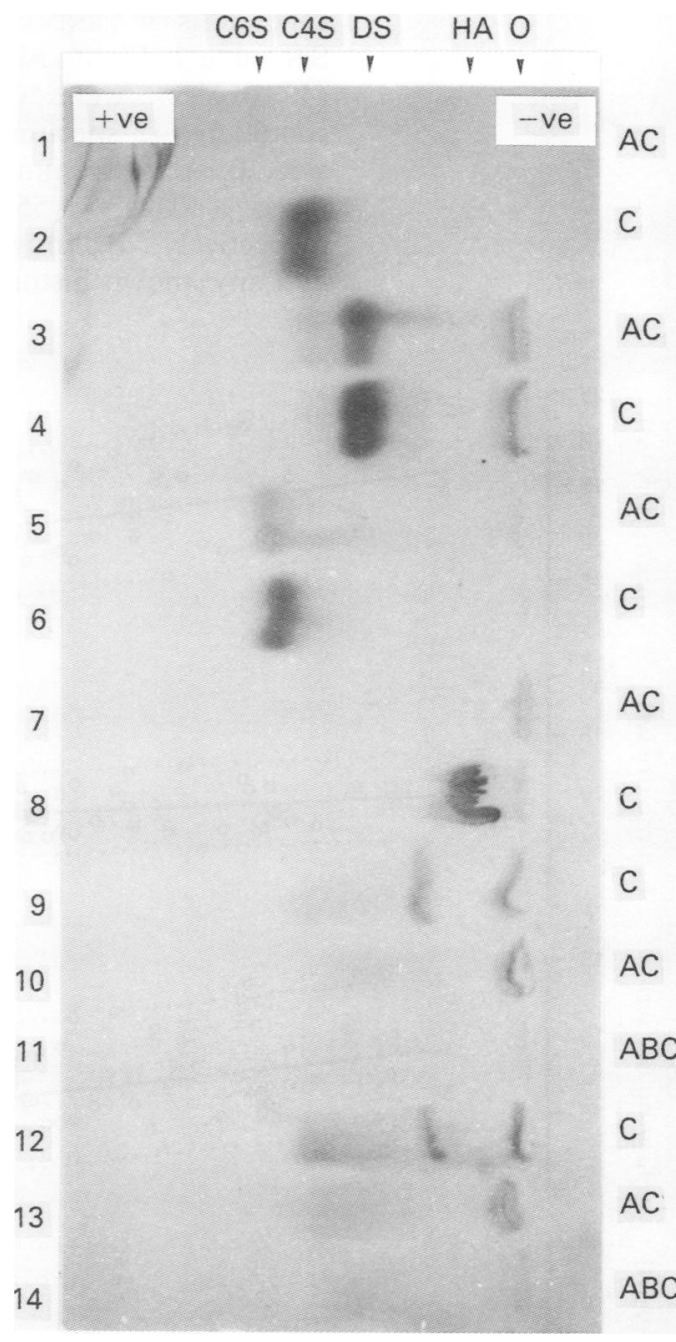

Figure 1 Cellulose acetate electrophoresis of glycosaminoglycans. Cadaver tendon specimens were digested with papain and the crude digest was subjected to electrophoresis at $45 \mathrm{~mA}$ for one hour in $0.3 \mathrm{M}$ calcium acetate buffer $\mathrm{pH}$ 7.2 and stained with $0.2 \%$ Alcian Blue $8 G X$ in $0.3 \%$ acetic acid 0.05 $\mathrm{M} \mathrm{MgCl}_{2}$. Glycosaminoglycan standards $(1.0 \mathrm{mg} / \mathrm{ml}$ each) of chondroitin-4-sulphate (C4S), chondroitin-6-sulphate (C6S), dermatan sulphate (DS) and hyaluronan $(H A)$ were obtained from Sigma. Some samples were digested with chondroitinase enzymes for four hours at $37^{\circ} \mathrm{C}$.

$O=$ origin. $A B C=$ chondroitinase $A B C$ digest .

$A C=$ chondroitinase $A C . C=$ undigested control.

Lanes 1 and 2, C4S; lanes 3 and 4,DS; Lanes 5 and 6, C6S; lanes 7 and 8, $H A$; lanes 9 to 11, 12 year old supraspinatus and lanes 12 to 14, 19 year old supraspinatus. 
Table 2 Glycosaminoglycan composition of human tendons

\begin{tabular}{|c|c|c|c|c|}
\hline Tendon specimens & $\begin{array}{l}G A G \\
\mu g / m g d w \\
(S D)\end{array}$ & $\begin{array}{l}U A \\
\mu g / m g d w \\
(S D)\end{array}$ & $\begin{array}{l}C S \\
\mu g / m g d w \\
(S D)\end{array}$ & $\begin{array}{l}D S \\
\mu g / m g d w \\
(S D)\end{array}$ \\
\hline $\begin{array}{l}\text { Supraspinatus (cadaver) } \\
\text { macroscopically 'normal' } \\
\text { 'ruptured' tendons } \\
\text { 'thin/degenerate' tendons } \\
\text { 'calcified' tendons } \\
\text { misc abnormalities }\end{array}$ & $\begin{array}{l}12 \cdot 3(4 \cdot 3) \\
10 \cdot 8(2 \cdot 6) \\
10 \cdot 9(4 \cdot 4) \\
12 \cdot 8(1 \cdot 4) \\
13 \cdot 3(3 \cdot 2)\end{array}$ & $\begin{array}{l}8 \cdot 5(1.9) \\
8 \cdot 7(1.5) \\
8 \cdot 4(2 \cdot 4) \\
8 \cdot 8(2 \cdot 3) \\
9 \cdot 9(1 \cdot 0)\end{array}$ & $\begin{array}{r}6 \cdot 9(2 \cdot 6) \\
5 \cdot 1(1 \cdot 3) \\
7 \cdot 2(0 \cdot 2) \\
8 \cdot 2(0 \cdot 6) \\
10 \cdot 4(0 \cdot 8)\end{array}$ & $\begin{array}{l}2 \cdot 5(1 \cdot 2) \\
1 \cdot 8(0 \cdot 2) \\
2 \cdot 2(0 \cdot 1) \\
2 \cdot 7(0 \cdot 4) \\
3 \cdot 4(0 \cdot 1)\end{array}$ \\
\hline $\begin{array}{l}\text { Common biceps (cadaver) } \\
\text { macroscopically 'normal' }\end{array}$ & $1 \cdot 2(0 \cdot 6)^{\star \star \star}$ & $5 \cdot 3(1 \cdot 9)^{\star \star \star}$ & $0 \cdot 2(0 \cdot 2)^{\star \star \star}$ & $0.8(0 \cdot 2)^{\star \star \star}$ \\
\hline $\begin{array}{l}\text { Supraspinatus biopsy } \\
\text { 'tendinitis' (cuff tear) } \\
\text { 'calcifying tendinitis' } \\
\text { fracture repair }\end{array}$ & $\begin{array}{r}13.7(4 \cdot 1) \\
9.9(3.9) \\
8.9(3 \cdot 7)\end{array}$ & $\begin{array}{l}18 \cdot 7(3.6)^{\star \star \star} \\
14.4(6 \cdot 5) \\
13.6(3.5)\end{array}$ & $\begin{array}{l}8 \cdot 4(2 \cdot 8)^{\star} \\
6 \cdot 8(2 \cdot 9) \\
4 \cdot 1(2 \cdot 8)\end{array}$ & $\begin{array}{l}3.8(1 \cdot 1)^{\star \star \star} \\
1.4(0.7) \\
3.3(0.5)\end{array}$ \\
\hline $\begin{array}{l}\text { Subscapularis biopsy } \\
\text { 'tendinitis' (cuff tear) } \\
\text { stabilisation operation } \\
\text { prosthetic replacement }\end{array}$ & $\begin{array}{l}9 \cdot 9(4 \cdot 1) \\
6 \cdot 1(1 \cdot 7)^{\star \star} \\
4 \cdot 5(2 \cdot 4)^{\star \star \star}\end{array}$ & $\begin{array}{c}12 \cdot 2(3 \cdot 7)^{\star \star} \\
6 \cdot 5(2 \cdot 0) \\
5 \cdot 7(3 \cdot 2)\end{array}$ & $\begin{array}{l}3.8(2 \cdot 2)^{\star \star \star} \\
1.9(0 \cdot 7)^{\star \star \star} \\
1.4(1 \cdot 5)^{\star \star \star}\end{array}$ & $\begin{array}{l}3 \cdot 7(1 \cdot 3)^{\star \star} \\
3 \cdot 1(0.8) \\
2 \cdot 6(0.6)\end{array}$ \\
\hline
\end{tabular}

$\mathrm{GAG}=$ total glycosaminoglycan; $\mathrm{UA}=$ uronic acid; $\mathrm{CS}=$ chondroitin sulphate; $\mathrm{DS}=$ dermatan sulphate.

Significance of difference from macroscopically normal supraspinatus tendon sample calculated by the Mann-Whitney test. ${ }^{\star}=\mathrm{p}<0.05,{ }^{\star \star} \mathrm{p}<0.01,{ }^{\star \star \star} \mathrm{p}<0.001$.

SPECIMENS OF TENDON

See table 1. Eighty two supraspinatus tendons (48 males) were obtained from cadaver shoulders, 42 specimens from the right hand side. Bilateral specimens were obtained from three cadavers (55, 59 and 91 years respectively). Although none of the cadavers had any known history of shoulder disorders,
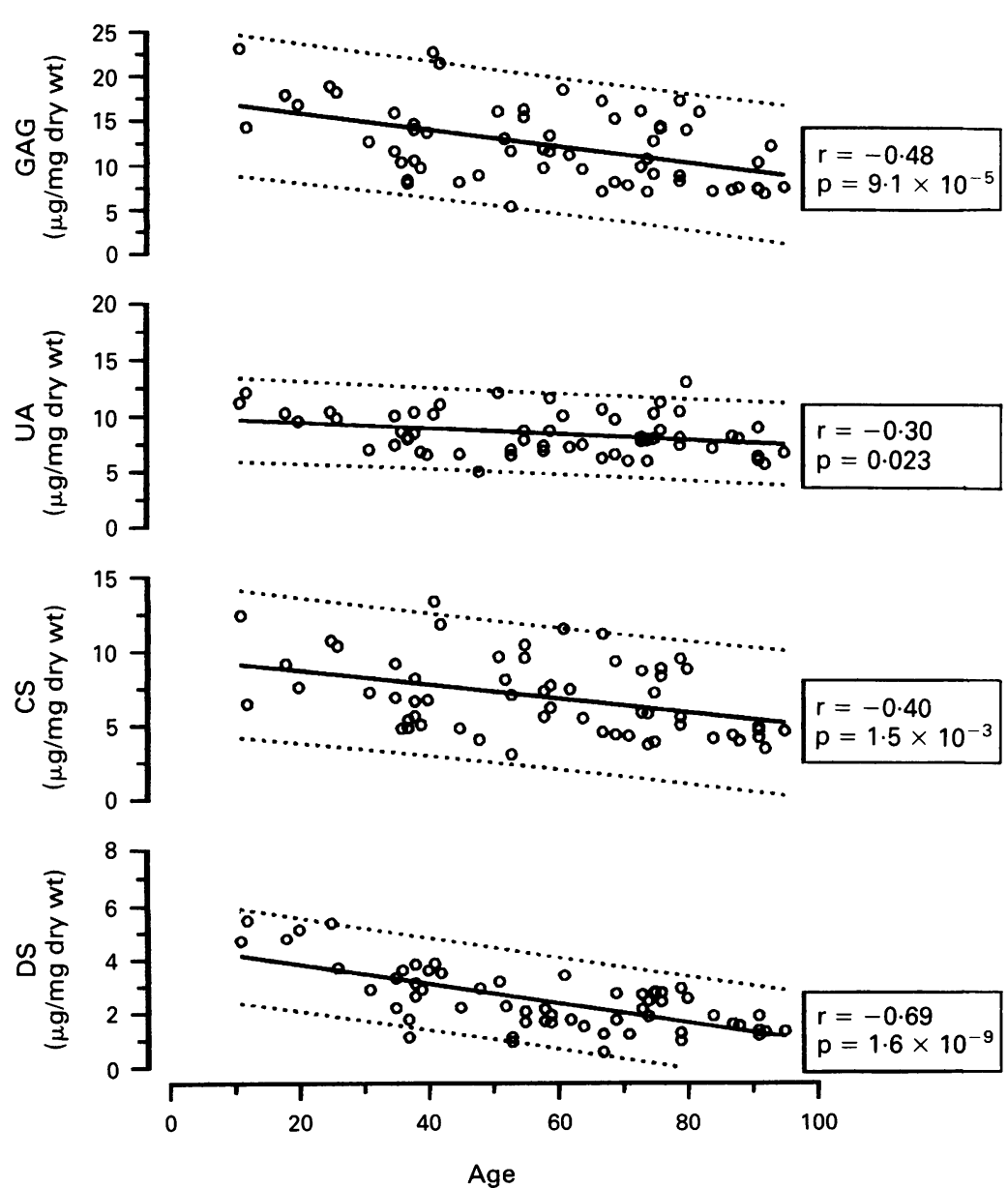

Figure 2 Changes in supraspinatus tendon glycosaminoglycans with age. Macroscopically Figure 2 Changes in supraspinatus tendon glycosaminoglycans with age. Macroscopically
normal cadaver supraspinatus tendons were digested with papain and duplicate aliquots of normal cadaver supraspinatus tendons were digested with papain and duplicate aliquots of
the supernatant were analysed for total sulphated glycosaminoglycan $(G A G)$, uronic acid $(U A)$, chondroitin sulphate (CS) and dermatan sulphate (DS). Data represent the mean of three papain digests of 60 tendons (age range 11-95, mean 58.1), expressed relative to the tendon dry weight. Dotted lines represent $95 \%$ confidence limits calculated by linear regression.
22 specimens had some form of macroscopic abnormality, according to the subjective assessment of one of us (MDC). Eleven specimens (five males) had some tendon fibre disruption, fraying or partial thickness tears and were designated 'ruptured', five specimens (two males) had a thin, atrophic appearance and were designated 'thin/degenerate'; five specimens (two males) contained white mineral deposits and were designated 'calcified'; three specimens were of an otherwise abnormal appearance and designated 'miscellaneous abnormalities'. One specimen was both 'ruptured' and 'calcified' and represented twice in the table. Eight 'normal' cadaver specimens (three males) between the ages of 19 and 88 were divided into approximately equal bone and muscle ends in a study of regional variation within the tendon.

All of the common biceps tendon specimens were of macroscopically normal appearance, with no indications of previous tendon pathology. Patients with rotator cuff tendinitis were treated conservatively for a variable period of at least three months before surgery, including at least one injection of corticosteroid. Specimens were taken from regions adjacent to the lesion and varied in size from less than 100 mg wet weight up to three grams.

\section{Results}

QUALITATIVE ANALYSIS OF

GLYCOSAMINOGLYCANS

Hyaluronan (HA) was the major glycosaminoglycan of the normal supraspinatus tendon, migrating as a trailing band slightly ahead of the commercial standard preparation and identified by digestion with specific glycosidase enzymes (fig 1).

Significant amounts of chondroitin sulphate (CS) and dermatan sulphate (DS) were also present in all of the normal supraspinatus tendons, although less abundant than hyaluronan. However, the diffuse nature of the bands, the small sample size and the low sensitivity of the technique prevented reproducible quantitative analysis by densitometric scanning analysis. In some specimens, a small amount of material was resistant to chondroitinase $\mathrm{ABC}$, which was possibly keratan sulphate (KS). See fig 1, lane 14. However, positive identification of this band by keratanase digestion was inconclusive, due to poor resolution and reproducibility of the digestion.

\section{QUANTITATIVE ANALYSIS OF}

GLYCOSAMINOGLYCANS

The quantitative data, expressed relative to the tendon dry weight, are shown in table 2 .

NORMAL CADAVER SUPRASPINATUS TENDON There was a significant decrease in both the sulphated glycosaminoglycan (GAG) content and the uronic acid content across the age range 11 to 95 years, although there was considerable variation within the sample (fig 2). 
Table 3 Regional variation of glycosaminoglycans in the supraspinatus tendon

\begin{tabular}{|c|c|c|c|c|c|}
\hline $\begin{array}{l}\text { Specimen } \\
\text { location }\end{array}$ & $\begin{array}{l}G A G \\
\mu g / m g d w \\
(S D)\end{array}$ & $\begin{array}{l}U A \\
\mu g / m g d w \\
(S D)\end{array}$ & $\begin{array}{l}C S \\
\mu g / m g d w \\
(S D)\end{array}$ & $\begin{array}{l}D S \\
\mu g / m g d w \\
(S D)\end{array}$ & $\begin{array}{l}C S / D S \\
\text { ratio } \\
(S D)\end{array}$ \\
\hline $\begin{array}{l}\text { Muscle end }(n=6) \\
\text { Bone end }(n=6)\end{array}$ & $\begin{array}{c}5 \cdot 5(2 \cdot 6) \\
11 \cdot 4(4 \cdot 1)^{\star}\end{array}$ & $\begin{array}{l}10 \cdot 5(3 \cdot 5) \\
13 \cdot 4(4 \cdot 1)^{\mathrm{ns}}\end{array}$ & $\begin{array}{l}2 \cdot 0(0 \cdot 7) \\
5 \cdot 4(2 \cdot 6)^{\star}\end{array}$ & $\begin{array}{l}2.0(0.5) \\
3 \cdot 2(0.9)^{\mathrm{ns}}\end{array}$ & $\begin{array}{l}1.0(0.3) \\
1.9(0.7)^{\star}\end{array}$ \\
\hline
\end{tabular}

GAG = total glycosaminoglycan; UA = uronic acid; CS = chondroitin sulphate; DS = dermatan sulphate.

ns $=$ not significant; ${ }^{\star}=p<0.05$, Mann-Whitney test.

The majority of the GAG was susceptible to digestion by chondroitinase $\mathrm{AC}$ and was identified as chondroitin sulphate (CS), although it was not possible to distinguish between chondroitin 4 sulphate $(\mathrm{C} 4 \mathrm{~S})$ and chondroitin 6 sulphate (C6S) by this method. A small proportion of the GAG was resistant to chondroitinase AC but susceptible to chondroitinase $\mathrm{ABC}$ and was identified as dermatan sulphate (DS). The concentration of both chondroitin sulphate and dermatan sulphate decreased significantly with age

- 'Normal' cadaver supraspinatus

'Abnormal' cadaver supraspinatus
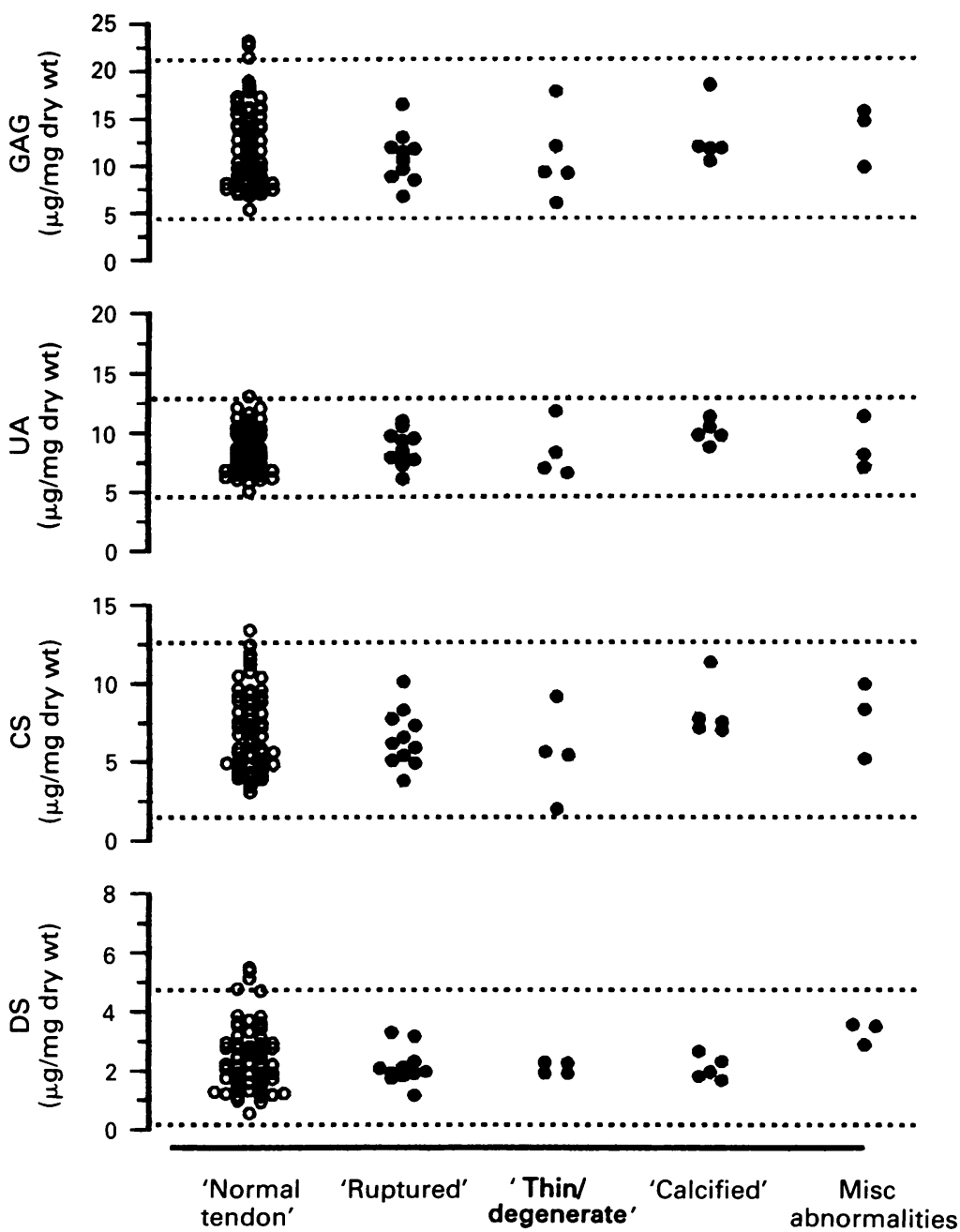

Figure 3 Normal versus macroscopically abnormal cadaver supraspinatus tendons. Cadaver supraspinatus tendons were digested with papain and duplicate aliquots of the supernatant were analysed for total sulphated glycosaminoglycan (GAG), uronic acid (UA), chondroitin sulphate (CS) and dermatan sulphate (DS). Data represent the mean of three papain digests of 60 macroscopically normal tendons (age range 11-95, mean $58 \cdot 1$ ), 11 'ruptured' tendons (age range 60-94, mean $81 \cdot 2$ ), 'calcified' tendons (age range 76-94, mean 82.0), 4 'thin/degenerate' tendons (age range 67-86, mean $78 \cdot 7$ ) and three tendons with 'misc abnormalities' (age range 43-84, mean 57-3).

Dotted lines represent the mean (2 SD). There was no significant difference between the normal and macroscopically abnormal specimens. (fig 2), although the relative proportion (CS/ DS) did not change (data not shown).

There was no significant difference between males and females, left and right shoulders and bilateral tendon specimens (data not shown). The same trends were observed when the data was expressed relative to the collagen content of the tendon (data not shown). There were, however, significant differences between the bone and muscle ends of the tendon (table 3 ). There was a greater concentration of GAG at the bone end of the tendon, the majority of which was chondroitin sulphate.

A proportion of the sulphated GAG extracted from the supraspinatus tendon was resistant to chondroitinase $A B C$, even after extended periods of digestion of up to five days (data not shown). However, after digestion with keratanase for 18 hours at $37^{\circ} \mathrm{C}$, only a small proportion (up to $40 \%$ ) of the dimethylmethylene blue dye-binding activity was eliminated. Consequently it was not safe to assume that all the glycosaminoglycan resistant to chondroitinase $\mathrm{ABC}$ was keratan sulphate (see KS ELISA data below).

The hyaluronan content was calculated from the uronic acid and GAG data, assuming that human tendon glycosaminoglycans contain the same proportion of uronic acid as animal glycosaminoglycans, as reported by Greiling. ${ }^{23}$ The mean (SD) hyaluronan content was 9.3 $(2 \cdot 8) \mu \mathrm{g} / \mathrm{mg}$ dry weight and there was no change with increasing specimen age (data not shown).

MACROSCOPICALLY 'ABNORMAL' CADAVER SUPRASPINATUS TENDONS

Although 22 of the cadaver tendons showed signs of previous tendon injury and pathology
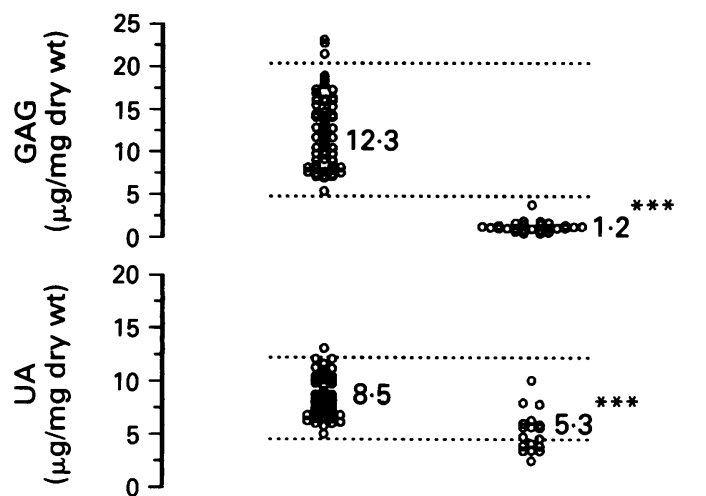

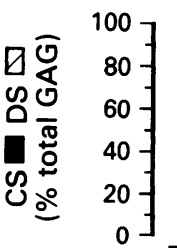

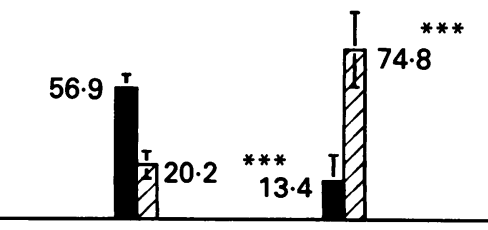

Supraspinatus Common biceps tendon tendon

Figure 4 Common biceps tendon versus supraspinatus tendon. Total sulphated glycosaminoglycan (GAG), uronic acid $(U A)$, chondroitin sulphate $(C S)$ and dermatan sulphate $(D S)$ were assayed as described. Data represent the mean of three papain digests of 60 macroscopically normal cadaver supraspinatus tendons (age range 11-95, mean $58 \cdot 1)$ and 26 normal cadaver common biceps tendons (age range 11-85). ${ }^{\star \star \star}=p<0.001$. 


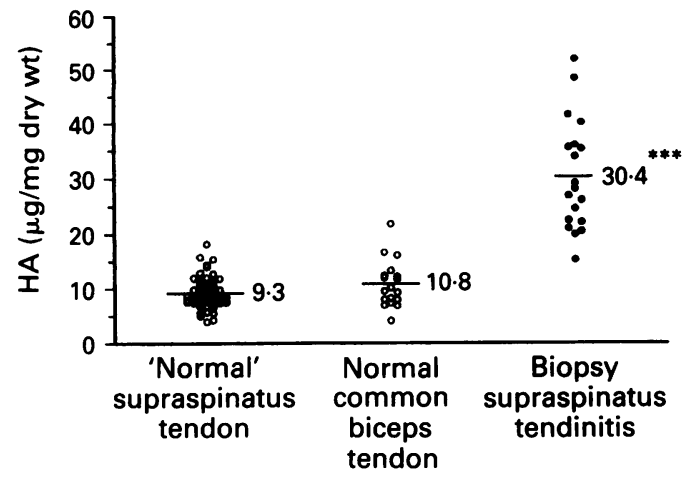

Figure 5 Hyaluronan of normal and pathological supraspinatus tendons. Hyaluronan was calculated from the $G A G$ and uronic acid content, assuming that sulphated $G A G$ 's contain $36 \%$ uronic acid and hyaluronan contain $45 \%$ uronic acid. Data shown for 60 macroscopically normal cadaver supraspinatus tendons (age range 11-95, mean 58.1), 18 normal cadaver common biceps tendons (age range 19-38, mean 55.3) and 19 supraspinatus specimens from patients with tendinitis (age range 38-80, mean $57 \cdot 6$ ).

There was no significant difference between normal cadaver supraspinatus and common biceps tendons, but there was a significant increase in hyaluronan in biopsy supraspinatus tendons form rotator cuff tendinitis. $\star \star \star x=0<0.001$

\section{- Cadaver supraspinatus \\ Biopsy supraspinatus}
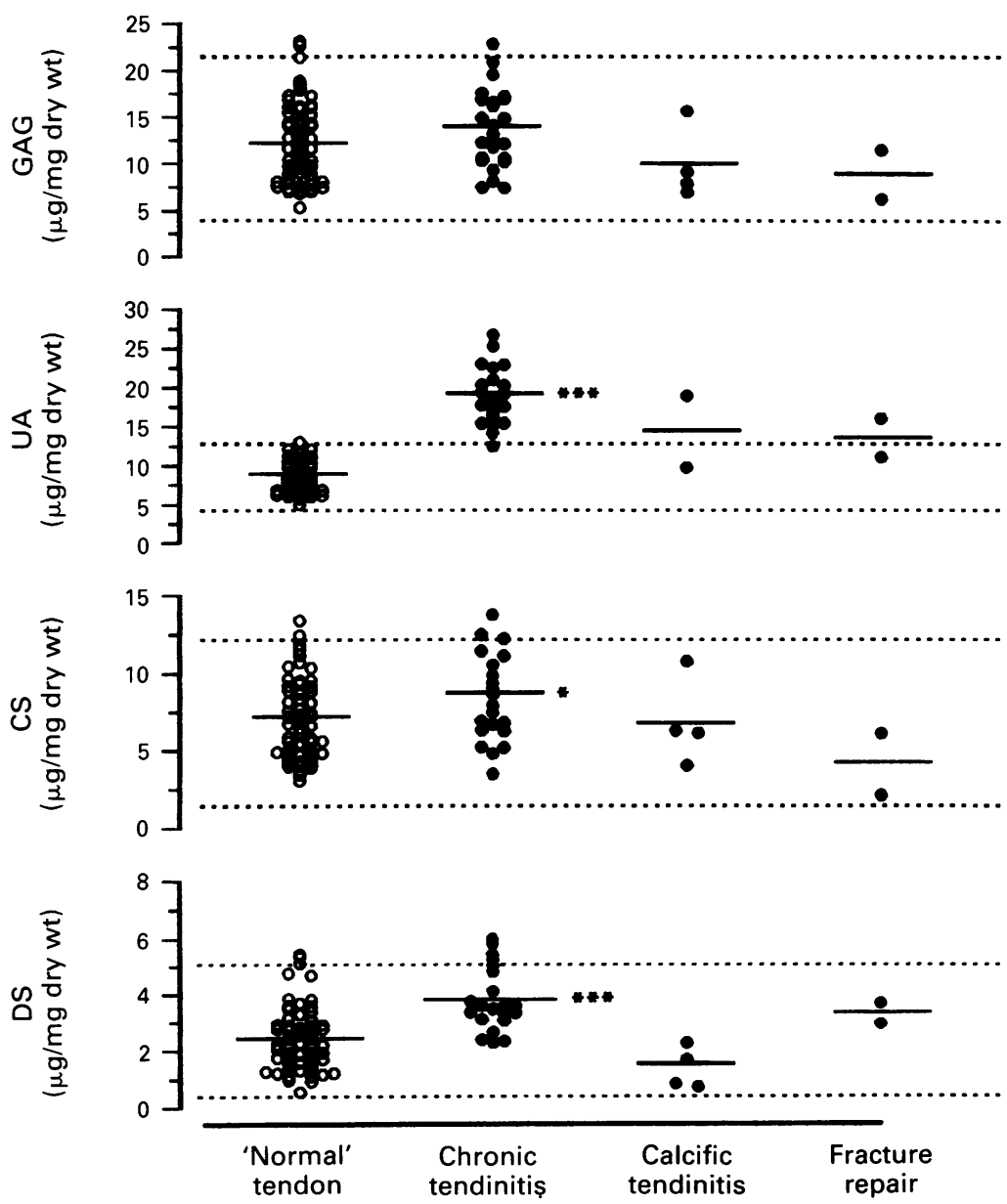

Figure 6 Glycosaminoglycans of biopsy specimens of supraspinatus tendon. Total sulphated glycosaminoglycan (GAG), uronic acid (UA), chondroitin sulphate (CS) and dermatan sulphate (DS) were assayed as described. Data shown for 26 specimens from patients with chronic tendinitis (age range 38-80, mean 59.5), four specimens with calcifying tendinitis (age range 47-63, mean 52.2) and two specimens from humeral fractures (25 and 78 years) and compared with 60 macroscopically normal cadaver supraspinatus tendons (age 11-95, mean 58.1).

Dotted lines represent the mean $(2 S D) .^{\star}=p<0.05,{ }^{\star * *}=p<0.001$. (including specimens which were designated 'ruptured', 'calcified' or 'thin and degenerate'), none were significantly different from the macroscopically normal supraspinatus tendon sample in respect of glycosaminoglycan content. All of the specimen values for each of the parameters (total GAG, UA, HA, CS, DS, and CS/DS ratio) were within the normal range, as defined by the normal sample mean (2 SD) (fig 3).

\section{COMMON BICEPS TENDON}

The common biceps tendons contained significantly less total GAG and uronic acid compared to the supraspinatus tendon $(p<0.001)$. In addition, the major sulphated glycosaminoglycan was dermatan sulphate, which comprised almost $75 \%$ of the total GAG (fig 4).

There was no significant change in the total GAG content of the biceps tendon with increasing specimen age and no change in the relative proportion of chondroitin sulphate and dermatan sulphate (data not shown). Although the total uronic acid content was significantly lower, the mean (SD) hyaluronan content was calculated to be $10 \cdot 8(4 \cdot 3) \mu \mathrm{g} / \mathrm{mg}$ dry weight, which was not significantly different from the normal supraspinatus tendon (fig 5). There was no significant change in hyaluronan with increasing specimen age, similar to the normal supraspinatus tendon sample (data not shown).

SUPRASPINATUS TENDONS FROM PATIENTS WITH CHRONIC ROTATOR CUFF TENDINITIS The total GAG content of biopsy specimens from supraspinatus tendinitis was not significantly different from normal cadaver supraspinatus tendons. However, there was a significant increase in uronic acid $(p<0.001)$, chondroitin sulphate $(p<0.05)$ and dermatan sulphate $(p<0.001)$. See fig 6 .

The mean (SD) hyaluronan content was $30.4(10.1) \mu \mathrm{g} / \mathrm{mg}$ dry weight, significantly greater than both 'normal' cadavers supraspinatus and common biceps tendons $(\mathrm{p}<0.001)$. See fig 5 .

Specimens of tendon from patients with calcifying tendinitis and from fracture repair operations were not significantly different from normal cadaver tendons, although there were few specimens and considerable variation within the samples.

SUBSCAPULARIS TENDONS FROM SHOULDER OPERATIONS

There were similarities between subscapularis tendons from patients with chronic tendinitis and the biopsy specimens of supraspinatus tendinitis. Although there was no significant difference in total GAG, there was a significant increase in both the uronic acid and dermatan sulphate content $(\mathrm{p}<0.01$ and $\mathrm{p}<0.001$ respectively). The chondroitin sulphate content, however, was significantly lower than that found in the normal supraspinatus tendon 

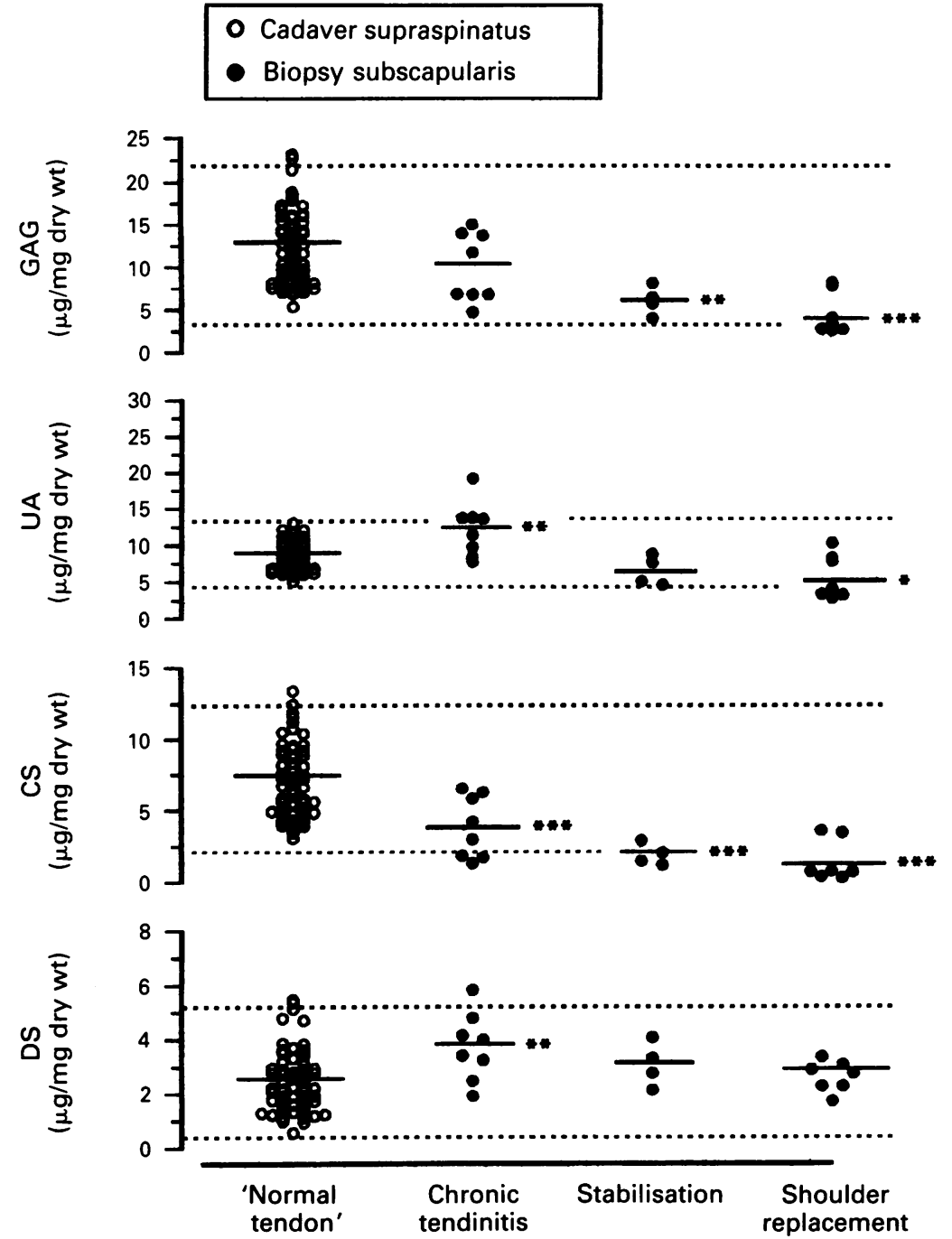

Figure 7 Glycosaminoglycans of biopsy specimens of subscapularis tendon. Total sulphated glycosaminoglycan (GAG), uronic acid (UA), chondroitin sulphate (CS) and dermatan sulphate (DS) were assayed as described. Data shown for eight specimens from patients with chronic tendinitis (age range 68-80, mean 73.4), four specimens from stabilisation operations (age range 19-31, mean 25.2) and seven specimens from shoulder replacement operations (age range 40-76, mean 57.3) and compared with 60 macroscopically normal cadaver supraspinatus tendons (age range 11-95, mean 58.1).

Dotted lines represent the mean (2SD). ${ }^{*}=p<0.05, * *=p<0.01, * * *=p<0.001$.

$(\mathrm{p}<0.001)$, although significantly greater (on average) than that found in the other subscapularis tendon biopsy specimens (fig 7).

Specimens from stabilisation operations and shoulder replacement operations had a similar GAG composition to the common biceps tendon, with a small quantity of predominantly dermatan sulphate proteoglycans and a very low chondroitin sulphate content. However these specimens are not necessarily representative of normal subscapularis tendons, as they are likely to have been subject to some abnormal stresses and/or pathology before the shoulder operation. Normal cadaver subscapularis tendons were not obtained.

Table 4 Keratan sulphate content of human tendons

\begin{tabular}{lll}
\hline Tendon specimens & $\begin{array}{l}\text { Mean age } \\
\text { (range) }\end{array}$ & $\begin{array}{l}\text { Keratan sulphate } \\
\mu g / m g d w ~(S D)\end{array}$ \\
\hline Normal cadaver supraspinatus $(\mathrm{n}=25)$ & $47(11-80)$ & $0 \cdot 43(0 \cdot 33)$ \\
Normal cadaver common biceps $(\mathrm{n}=8)$ & $61(41-73)$ & $0 \cdot 04(0 \cdot 05)^{\star \star \star}$ \\
Biopsy supraspinatus tendinitis $(\mathrm{n}=6)$ & $61(45-80)$ & $0 \cdot 18(0 \cdot 05)^{\text {ns }}$ \\
\hline
\end{tabular}

Papain digests were analysed for keratan sulphate using the 5-D-4 monoclonal antibody ELISA. ns $=$ not significant, ${ }^{\star \star \star} \mathrm{p}<0.001$ Mann-Whitney test.
KERATAN SULPHATE ANALYSIS

The presence of keratan sulphate in the supraspinatus tendon matrix was confirmed by ELISA using the 5D4 monoclonal antibody (table 4).

There was no significant change in keratan sulphate content with age, although there was a large degree of variation in the sample. In addition, there was no significant difference in the diseased specimens of supraspinatus tendon, although a small number of specimens were analysed. In contrast, very little or no keratan sulphate was detected in specimens of normal common biceps tendon. However, it is acknowledged that the ELISA technique may underestimate the true keratan sulphate content of the tendons, as the assay is specific for a single epitope, which might be hidden or only selectively expressed in some forms of keratan sulphate.

PROTEOGLYCAN ANALYSIS

Almost $90 \%$ of the tendon proteoglycans were solubilised after two sequential extractions ( 48 and 24 hours respectively) with $4 \mathrm{M}$ guanidine

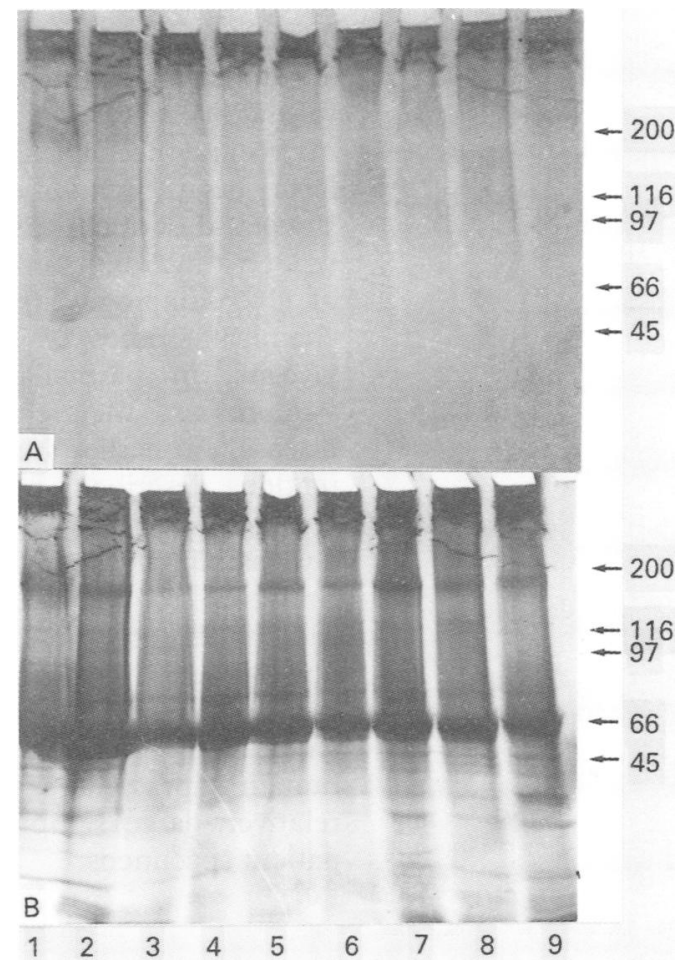

Figure 8 4-15\% SDS PAGE of supraspinatus tendon proteoglycans. Macroscopically 'normal' cadaver supraspinatus tendons were extracted with $4 \mathrm{M} \mathrm{GuHCl}$ (containing protease inhibitors) and the soluble proteoglycans were precipitated with nine volumes of ethanol (containing $1 \%$ potassium acetate) and taken up in reducing SDS sample buffer. $20 \mu$ of each sample (containing between 5 and $10 \mu \mathrm{g}$ of sulphated $G A G$ ) was subject to electrophoresis at $200 \mathrm{~V}$ for 40 minutes, washed extensively to remove SDS and stained with $0 \cdot 2 \%$ Alcian Blue 8GX followed by Coomassie Brilliant Blue G250. Lanes 1 to 9 contain extracts from specimens of 92, 84, 71, $67,55,41,38,26$ and 12 years old respectively. $(A)$ Alcian Blue $8 G X$ stained gel. (B) The same gel stained with Coomassie Brilliant Blue G250. Three alcian blue stained proteoglycans were visible in all of the tendon specimens; a large proteoglycan at the gel interface and two small proteoglycans of approximate molecular weight 220 $\mathrm{kD}$ and between $80-130 \mathrm{kD}$. A large number of proteins were visible in all of the tendon extracts. No qualitative difference was observed between specimens. 
and there was no significant difference in the proteoglycan solubility between the different tendon samples (data not shown). The proteoglycans were taken up in SDS sample buffer and analysed by reducing $4-15 \%$ gradient SDS-PAGE. Three species of proteoglycan were seen in supraspinatus tendons from across the age range (fig 8).

Two diffuse and lightly stained bands, with relative molecular weights of approximately $100 \mathrm{kD}$ (range $80-130 \mathrm{kD}$ ) and $220 \mathrm{kD}$, were tentatively identified as the small proteoglycans decorin and biglycan respectively, on the basis of previously published data. ${ }^{27}$ The proteoglycan at the gel interface, excluded by its size from entering the polyacrylamide matrix, was probably a large proteoglycan such as aggrecan, which has been identified by similar methods in some animal tendons and more recently in the human tibialis anterior tendon. ${ }^{28} \mathrm{~A}$ large number of proteins were present in each of the tendon extracts (fig $7 \mathrm{~b}$ ), although no qualitative difference was observed with age.

\section{Discussion}

The vulnerability of the rotator cuff tendons to degeneration and rupture has been ascribed to a number of factors, including a reduction in vascularity, age related changes of the tendon matrix and mechanical trauma. Although there have been a number of histopathological studies describing degenerative changes in tendons, including an abnormal accumulation of glycosaminoglycans, there have been few chemical studies of human tendon proteoglycans, in particular of the rotator cuff tendons and their changes with age and in disease. An earlier study of the supraspinatus tendon described histological evidence of chemical degradation of both collagen and glycosaminoglycans with increasing age. ${ }^{29}$ Brewer's study, however, was of just three cadaver tendons and cannot be presumed to be illustrative of the ageing process. This study is the first major qualitative and quantitative study of the glycosaminoglycans of the adult human supraspinatus tendon matrix, from a relatively large sample of both cadaver and patient specimens.

AGE RELATED CHANGES OF NORMAL ADULT HUMAN TENDONS

We have described a significant reduction in both chondroitin sulphate and dermatan sulphate with increasing specimen age, but no change in the content of hyaluronan. Similar changes in glycosaminoglycan composition have been documented in ageing animal tendons. ${ }^{15}$ These changes correspond with a reduction in the cellularity of the tendons and imply a reduction in the cellular activity of the tendons with age, although we could find no direct correlation between the DNA content and the GAG content in these specimens (unpublished observations).

The maintenance of the tendon matrix is an important function of the tenocytes and has important consequences for the ability of the tissue to resist mechanical forces and to repair in response to tendon injury. A reduced vascular perfusion in the 'critical zone' of the supraspinatus tendon may be in part responsible for this reduction in proteoglycan synthetic activity, although it is also possible that senescence of the cell population is a significant factor. In this respect it is interesting that the proliferative activity of adult human tenocytes is not significantly affected by age. ${ }^{31}$ It is possible that the tenocytes are less synthetically active and consequently their ability to maintain the tendon matrix may be significantly reduced. Future studies of tenocytes isolated from ageing adult supraspinatus tendons are required to address this question.

WHAT FACTORS INFLUENCE SUPRASPINATUS TENDON GLYCOSAMINOGLYCAN COMPOSITION? There was a large degree of variation in the sample, with some older tendons containing as much glycosaminoglycan as younger tendons, which suggests that factors other than age have an influence on the glycosaminoglycan content of the tendon. For example, the glycosaminoglycan composition may be closely linked to the levels of physical activity of an individual, as it has been shown that immobilisation causes a reduction in glycosaminoglycan content and exercise can reverse the agerelated decline in glycosaminoglycan content of the rat patellar tendon. ${ }^{15}$ It is possible that the differences in glycosaminoglycan content may reflect variations in the diameters of the tendon collagen fibres, as proteoglycans and their constituent glycosaminoglycans have been shown to influence collagen fibrillogenesis in vitro and there is a strong correlation of glycosaminoglycan content with the diameter of collagen fibrils in vivo. ${ }^{32}$ For example, large diameter fibrils $(>150 \mathrm{~nm})$ are found in tissues containing predominantly dermatan sulphate, small diameter fibrils $(<60$ $\mathrm{nm}$ ) are found in tissues rich in hyaluronan and intermediate diameter fibres (60 to $150 \mathrm{~nm}$ ) are found in tissues rich in chondroitin sulphate. A typical flexor tendon contains a low concentration of dermatan sulphate and has a high proportion of large diameter collagen fibres. In addition, the mean collagen fibril diameter increases with maturation and correlates with a decrease in dermatan sulphate and an increase in the tensile strength of the tissue. ${ }^{33}$ Consequently, the glycosaminoglycan composition may have important consequences for the organisation and structural strength of the supraspinatus tendon matrix and suggests the possibility that the supraspinatus tendon has a smaller mean diameter of collagen fibrils compared with a typical flexor tendon.

THE NORMAL SUPRASPINATUS TENDON CONTAINS REGIONS OF FIBROCARTILAGE An important observation in this study was the apparent fibrocartilaginous nature of the 
supraspinatus tendon, compared to a typical flexor tendon. The characteristic features of animal tendon fibrocartilage include an increased glycosaminoglycan content, consisting of a high proportion of chondroitin sulphate proteoglycans, a small proportion of keratan sulphate and an increased cell density. ${ }^{34}$ Preliminary analysis of the soluble proteoglycans of the supraspinatus tendon indicated the presence of both large and small proteoglycans. The small proteoglycans have been tentatively identified as biglycan and decorin and the large proteoglycan(s) as aggrecan or versican. The presence of keratan sulphate may indicate that the small proteoglycan fibromodulin is also present. Biglycan and aggrecan are normally restricted to compressed regions of animal tendons and their presence would appear to confirm the fibrocartilaginous nature of the human supraspinatus tendon.

Compressive forces are important for the development and maintenance of tendon fibrocartilage. For example, a rapid loss of chondroitin sulphate can be induced by translocation of the flexor digitorum profundus tendon to a different site. ${ }^{16}$ In addition, fibrocartilaginous regions of adult bovine tendons require cycles of compressive loading to maintain large proteoglycan expression in explant culture. ${ }^{35}$ The exact mechanism by which the tendon cells sense their mechanical environment is not yet known, but a variety of factors such as changes in the cell shape, mechanical deformation of the cytoskeleton, reduced vascular supply and nutrient flow, low oxygen tension and soluble factors such as TGF- $\beta$ have all been implicated. ${ }^{36}$ However, some genetic influence is also likely, since the morphological appearance of fibrocartilaginous regions precedes the onset of weight bearing on fetal bovine flexor digitorum profundus tendons. ${ }^{35}$

\section{IMPLICATIONS FOR TENDON PATHOLOGY} It is probable that a fibrocartilaginous structure, which is normally associated with a more open, basket-weave pattern of smaller diameter collagen fibres ${ }^{35}{ }^{36}$ will have important consequences for the mechanical strength of the supraspinatus tendon, resulting in a reduced capacity to resist tensional forces. As yet there have been no studies demonstrating a difference in the tensile properties of fibrocartilage in animal tendons. In addition, the fibrocartilaginous composition may also have important consequences for the wound healing potential of the tendon. In animal tendons, these regions are relatively avascular and levels of DNA synthesis are lower compared to adjacent tension bearing regions. ${ }^{37}$ An avascular 'critical zone' is also proposed in the supraspinatus tendon and in theory these regions have a reduced capacity to repair. However, the fibrocartilaginous region of the canine flexor digitorum profundus tendon demonstrated a more rapid return of strength after injury than the adjacent tension bearing region. ${ }^{38}$ The difference in response may be related to the specific magnitude and/or orientation (compression, shear or tension) of the mechanical forces acting on the respective repair sites. The relevance of these studies to adult human supraspinatus tendons is open to question and future studies are required to examine the repair response in ageing human tendons at different anatomical sites.

It has been previously proposed that 'fibrocartilaginous transformation' is a degenerative event which may pre-dispose the tendon to rupture. ${ }^{19}$ This study has shown that the biochemical profile of tendon fibrocartilage is a normal feature of the supraspinatus tendon, which is more pronounced at the bone end compared with the muscle end of the tendon. The anatomical disposition of the supraspinatus suggests that for part of its length, in particular towards the bone insertion, the tendon is compressed against the head of the humerus below or squeezed against the acromium and/or coraco-humeral ligament above, particularly when the arm is elevated. The subscapularis tendon is less often affected by 'tendinitis' than the supraspinatus tendon and it may be significant that subscapularis tendons not affected by tendinitis were similar in composition to a normal flexor tendon which experiences predominantly tensional forces. It is possible that the subscapularis tendon, which is located in the anterior region of the rotator cuff complex, does not normally experience significant compressive and/or shear forces. In contrast, subscapularis specimens from patients with chronic tendinitis had a significantly higher concentration of chondroitin sulphate. This may be caused by an altered pattern of mechanical forces on the tendon before rupture. Impingement of the supraspinatus may be a principal cause of rotator cuff tears ${ }^{8}$ and it might be expected that the tendons of certain individuals may be disposed to a greater degree of compression than other specimens. This may be due to either the anatomical shape and slope of the acromium or to particular repetitive and/or overhead activities related to certain sports and occupations. Consequently, the interaction of functional requirements, extrinsic mechanical factors and anatomical variation may affect the intrinsic cell matrix response of the tenocytes in the rotator cuff tendons in the shoulder.

\section{INFLAMMATION AND REPAIR IN DEGENERATIVE} 'TENDINITIS'

This study has also shown that the degeneration of tendons affected by chronic tendinitis is not accompanied by a decrease in the glycosaminoglycan content, as described in other pathological situations, such as, articular cartilage fibrillation and osteoarthritis. ${ }^{39}$ In contrast, the principal changes are a substantial increase in hyaluronan accompanied by an increase in dermatan sulphate and, to a lesser degree, of chondroitin sulphate. These changes are consistent with the inflammatory phase of wound healing, with new matrix synthesis produced in response to acute tendon injury. ${ }^{17}$ 
An increased concentration of the small dermatan sulphate proteoglycan decorin is associated with the increased surface area of newly synthesised, small diameter collagen fibres ${ }^{11}{ }^{30}$ and hyaluronan is increased in situations of cell migration, proliferation and repair activity. ${ }^{40}$ This cell activity is associated with a fibroproliferative response and scar formation, as demonstrated by us in a recent study showing an increase in the proportion of type III collagen in the tendon matrix. ${ }^{+1}$

In conclusion, rotator cuff tendinitis is likely to have a multi-factorial aetiology, with a combination of intrinsic, degenerative changes exacerbated by extrinsic factors such as impingement and trauma. Whatever the cause, the chronic nature of the pathology is essentially a problem of poor tissue repair, as the structural integrity of the tendon depends on the ability of the ageing tendon cells to respond to the tendon injury and to replace and/or maintain the normal tendon matrix. It is a source of some controversy whether the healing response of the supraspinatus tendon can repair the tendon defect, which may depend critically on factors such as the size and site of the lesion. This study has demonstrated that human tendons are heterogeneous in biochemical composition and that these differences have important implications for the tendon cell-matrix response to injury and mechanical influences. These are important factors that need to be taken into consideration if we are to improve our understanding and treatment of chronic tendon pathology such as rotator cuff tendinitis.

This study was supported by a grant from the Arthritis and Rheumatism Research Council. The assistance of Dr Mike Bayliss and Ms Paula Hazell, who performed the keratan sulphate analysis, is gratefully acknowledged.

1 Wood P H N, Sturrock A W, Badley E M. Soft tissue rheumatism in the community. Clin Rheum Dis 1979; 5 743-53.

2 Chard M D, Hazleman B L. Shoulder disorders in the elderly (a hospital study). Am Rheum Dis 1987; 46 $486-7$.

3 Codman E A. The Shoulder. Boston: Todd Co, 1934.

4 Chard M D, Satelle L. M, Hazleman B L. The long term outcome of rotator cuff tendinitis-a review study. $\mathrm{Br}$ Rheum 1988; 27: 385-90.

5 Fearnley M E, Vadasz I. Factors influencing the response of lesions of the rotator cuff of the shoulder to local steroid injections. Amn Phys Med 1969; 10: 53-63.

6 McMaster P E. Tendon and muscle repair: clinical and experimental studies of the course and location of subcutaneous ruptures. F Bone foint Surg 1933; 15A 705-22.

7 MacNab I. Rotator cuff tendinitis. Ann Royal Coll Surg Eng 1973; 52: 271-87.

8 Neer C S. Impingement lesions. Clin Orthop 1983; 173: $70-77$.

9 Rothman R H, Parke W W. The vascular anatomy of the rotator cuff. Clin Orthop Rel Res 1965; 41: 176-86.

10 Hardingham T E, Fosang A J. Proteoglycans: many forms and many functions. FASEB 7 1992; 6: 861-70.

11 Scott J E. Proteoglycan-fibrillar collagen interactions. Biochem f 1988; 252: 313-23.

12 Ruoslahti E. Proteoglycans in cell regulation. $f$ Biol Chem 1989; 264: 13369-72.

13 Riemersma D J, de Bruyn P. Variations in cross-sectional area and composition of equine tendons with regard to their mechanical function. Res $V c t$ Sci 1986; 41: 7-13.
14 Ippolito E, Natali P G, Postacchini F, Accini L, de Martino C. Morphological immunological and biochemical study of rabbit achilles tendon at various ages. $\mathcal{f}$ Bone ft Sur $_{\mathrm{t}}$ 1980; 62A: $583-98$.

15 Vailas A C, Pedrini V A, Pedrini-Mille A, Holloszy J (). Patellar tendon matrix changes associated with aging and voluntary exercise. 7 Appl Phwsiol 1985; 58: 1572-6.

16 Gillard G C, Reilly H C, Bell-Booth P G, Flint M H. The influence of mechanical forces on the glycosaminoglycan content of the rabbit flexor digitorum profundus tendon. Conn Tissuc Ris 1979; 7: 37-46.

17 Flint M. Interrelationships of mucopolysaccharide and collagen in connective tissue remodelling. 7 Embroyl kixp Morph 1972; 27: 481-95.

18 Kannus P, Jozsa L. Histopathological changes preceding spontaneous rupture of a tendon. F Bonc Foint Surg 1991; 73A: 1507,25

19 Chard M D, Cawston 'T E, Riley G P, Gresham G A Hazleman B L. Rotator cuff degeneration and lateral epicondylitis - a comparative histological study. Ann Rheum Dis 1093: 53: 30-34.

20 Bergman I, Loxley R. Two improved and simplified methods for the spectrophotometric determination of hydroxyproline. Anal Biochem 1963; 35: 1961-5.

21 Farndale R W, Buttle D J, Barrett A J. Improved quantitation and discrimination of sulphated glycosaminoglycans by use of dimethylmethylene blue. Bioch Bioph's Acta 1986; 883; 173-7.

22 Bitter T, Muir H M. A modified uronic acid carbazole reaction. Anal Biochem 1962; 4: 330-4.

23 Greiling H. Glycosaminoglycans. In: Curtius H Ch, Roth $\mathrm{M}$, eds. Citinical biochemistry-principles and methods. Berlin, New York: De Gruyter, 1974: 944-71.

24 Caterson B, Christner J E, Baker J R. Identification of a monoclonal antibody that specifically recognises corneal and skeletal keratan sulphate. $\mathcal{F}$ Biol (Chcm 1983; 258: 8838-54.

25 Laemmli U K, Favre M. Maturation of the head of bacteriophage T4. F Mol Biol 1973; 80: 575-99.

26 Wall R S, Gyr T J. Alcian blue staining of protenglycans in polyacrylamide gels using the "critical electrolyte concentration" approach. Anal Biochem 1988; 175: concen $298-9$.

27 Rosenberg L C, Choi H U, Tang L-H, Johnson T L, Pal $\mathrm{S}$. Isolation of dermatan sulfate proteoglycans from mature bovine articular cartilages. F Biol ( hicm 1985; 260: $6304-13$.

28 Vogel K G, Ördög A, Pogany G, Oláh J. Proteoglycans in the compressed region of human tibialis posterior tendon and in ligaments. F Orthop Res 1993; 11: 68-77.

29 Brewer B J. Aging of the rotator cuff. Am F Sports Med 1979; $7(2): 102-10$

30 Scott J E, Orford C R, Hughes E W. Proteoglycan-collagen arrangements in developing rat tail tendon. Biochem $y$ 1981; 195: 573-81

31 Chard M I), Wright J K, Hazleman B L. Isolation and growth characteristics of adult human tendon fibroblasts. Ann Rhcum Dis 1987; 46: 385-90.

32 Merrilees .M J, Tiang K . M, Scott L. Changes in collagen fibril diameters across artery walls including a correlation with glycosaminoglycan content. Comn Tiss Res 1987; 16: $237-5 \%$.

33 Parry D A D, Barnes G R G, Craig A S. A comparison of the size distribution of collagen fibrils in connective tissues as a function of age and a possible relation between fibril size distribution and mechanical properties. Proc $R$ Soc Lond B 1978; 203: 305-21

34 Evanko S P, Vogel K G. Ultrastructure and proteoglycan composition in the developing fibrocartilaginous region of bovine tendon. Matrix 1990; 10: 420-36.

35 Koob T J, Clark P E, Hernadez D J, Thurmond F A, Vogel $\mathrm{K}$ G. Composition loading in vitro regulates proteoglycan sunthesis by tendon fibrocartilage. Arch Biochem Bioply' 1992; 298: 303-12.

36 Vogel K G, Koob T J. Structural specialisation in tendons under compression. Int Rev Cvtol 1989; 115: 267-93.

37 Abrahamsson S O. Matrix metabolism and healing in the flexor tendon. Experimental studies on rabbit tendon. Scand F Plast Surg Hand 1991; 25 (suppl 23): 1-51.

38 Nessler J P, Amadio P C, Berglund L J, An K-N. Healing of canine tendon in zones subjected to different mechanical forces. F Hand Surg 1992; 17B: 561-8.

39 Stockwell R A. Cartilage failure in osteoarthritis: relevance of normal structure and function. A review. Clin Anat 1991; 4: 161-91.

40 Burd D A R, Greco R M, Regauer S, Longaker M T, Siebert $\mathrm{J}$ W, Garg H G. Hyaluronan and wound healing: A new perspective. Brf Plast Surg 1991; 44: 579-84.

41 Riley G P, Harrall R L, Constant C R, Chard M D, Cawston $\mathrm{T}$ E, Hazleman B L. Tendon degeneration and chronic T E, Hazleman B L. Tendon degeneration and chronic shoulder pain: changes in the collagen composition of the
human rotator cuff tendons in rotator cuff tendinitis. $A m n$ human rotator cuff tendons in 